\title{
Noninvasive Method of Force Estimation of Lower Limb Muscles in Cricket Fast Bowlers: Can This be an Application to Minimize Potential Injury?
}

\author{
Subham Badhyal ${ }^{1}$, Harpreet Kaur ${ }^{2}$, Abhishek K Tiwari ${ }^{3}$, Sidak Dhillon ${ }^{4}$, Keddin Alwar Thiagarajan ${ }^{5}$, Sivaraman Arumugam $^{6}$,
} Mandeep S Dhillon ${ }^{7}$

\begin{abstract}
Background: Musculoskeletal modeling uses experimental data (motion capture data, both kinematics and kinetics) as an input to estimate the internal loading conditions; this noninvasive method is being validated by the research community for various activities and sports. With the heightened interest in Cricket in the Indian subcontinent, lower limb injuries are being increasingly documented in fast bowlers in cricket. The knowledge of muscular loading in lower limb during fast bowling has the potential to provide more insight into the mechanism of injury and help in prevention and management of the injury.

Aims: We aimed to document the muscle and joint contact forces in lower limbs of fast bowlers to see if these could act as indirect determinants to prognosticate injury potential.

Materials and methods: Experimental gait data from four Indian male state-level fast bowlers with no musculoskeletal injury in the past 6 months were collected in a gait laboratory. Both kinematic and kinetic data were collected using Plug-in Gait marker protocol. Inverse kinematics and inverse dynamics were used on the processed data to predict the internal joint loads. Static optimization was then used to estimate the muscle loading in the lower limb using OpenSim.

Results and discussion: Peak front foot vertical ground reaction forces were between 5.86 and 9.10 BW (body weight). Based on the kinematic and kinetic input, the forces induced in the muscles and their loading pattern for front lower limb were determined. This loading information (muscle force pattern) could act as an input for subject-specific interventions.

Conclusion: This study provides an insight to understand the lower limb muscular loading in fast bowlers. This information can be utilized by the sport scientists and medical professionals to access the injury in a more subject-specific manner. This quantitative information on lower limb loading at various joints will help in designing efficient strengthening and rehabilitation protocol for individual fast bowler.

Keywords: Bowling kinematics, Cricketinjury prevention, Fast bowler, Force estimation.

Journal of Postgraduate Medicine, Education and Research (2020): 10.5005/jp-journals-10028-1410
\end{abstract}

\section{INTRODUCTION}

Musculoskeletal modeling is a noninvasive method to understand the effect of motion and external forces on the musculoskeletal systems. ${ }^{1,2}$ The modeling process involves the approximation of mass plus inertial and geometrical properties of the skeletal system to represent a rigid body/biomechanical model. ${ }^{2}$ The biomechanical model consists of segments, connected by joints representing the anatomical structures and motion. The muscles connect segments and actuate the segments and joints to represent the resulting motion. The muscle and joint contact forces can be calculated using inverse dynamics, or the muscle and external forces are estimated using the forward dynamics approach. $^{2}$ The information obtained can provide more insight and knowledge about how individual muscles contribute toward the dynamic activity under investigation. This information on the musculoskeletal loading is not possible experimentally and noninvasively. This technique uses experimental data (motion capture data, both kinematics and kinetics) as an input to estimate the internal loading conditions and is being validated by research community for various activities and sports. ${ }^{1,2}$

The expansion of cricket internationally, with huge audiences and player volumes in the subcontinent, brings with it the potential
${ }^{1}$ TOPS, Sports Authority of India, New Delhi, India

${ }^{2}$ Department of Electronics and Communication Engineering, Guru Nanak Institutions of Technical Campus, Telangana, India

${ }^{3}$ Department of Applied Mechanics, Motilal Nehru National Institute of Technology, Allahabad, Uttar Pradesh, India

${ }^{4}$ Chennaiyin FC, Chennai, India

${ }^{5,6}$ Department of Arthroscopy and Sports Medicine, Sri Ramachandra Institute of Higher Education and Research, Chennai, Tamil Nadu, India

${ }^{7}$ Department of Orthopaedics, Postgraduate Institute of Medical Education and Research, Chandigarh, India

Corresponding Author: Subham Badhyal, TOPS, Sports Authority of India, New Delhi, India, Phone: +91 9815415396, e-mail: subhambadhyal@gmail.com

How to cite this article: Badhyal S, Kaur H, Tiwari AK, et al. Noninvasive Method of Force Estimation of Lower Limb Muscles in Cricket Fast Bowlers: Can This be an Application to Minimize Potential Injury? J Postgrad Med Edu Res 2020;54(4):236-240.

Source of support: Nil

Conflict of interest: None

(0) The Author(s). 2020 Open Access This article is distributed under the terms of the Creative Commons Attribution 4.0 International License (https://creativecommons. org/licenses/by-nc/4.0/), which permits unrestricted use, distribution, and non-commercial reproduction in any medium, provided you give appropriate credit to the original author(s) and the source, provide a link to the Creative Commons license, and indicate if changes were made. The Creative Commons Public Domain Dedication waiver (http://creativecommons.org/publicdomain/zero/1.0/) applies to the data made available in this article, unless otherwise stated. 
for significant injury. Fast bowlers, due to the nature of their game, bring huge amounts of strain to areas that are prone to injury, especially the lower back and lower limb. Lower limb injuries are known to contribute substantially to the injury in fast bowlers. ${ }^{3}$

Stretch reported an injury incidence of $49 \%$ in first league, provincial, and schoolboy cricketers in South Africa. ${ }^{4,5}$ The injury is reported predominantly in bowlers with incidence of $42 \%$ in first league and provincial cricketers and $47.4 \%$ in schoolboy cricketers. ${ }^{4,5}$ The incidence of injury in bowlers in England is more than the allrounders, batsmen, or wicketkeepers (as reported by Leary 2000), with an incidence of 57.4 days per 1,000 days of acute injury in professional players. ${ }^{6}$ The fast bowlers reportedly sustained more muscle/tendon injuries in comparison to other players. ${ }^{6}$

Foster et al. in a study of 82 fast bowlers reported that fast bowlers who bowled in more than 17 matches are at a higher risk of stress fractures and soft tissue injury. ${ }^{7}$ Subsequently, based on the research conducted on the prevalence of injury in fast bowlers, The England and Wales Cricket Board (ECB) issued guidelines in 1992 on the number of balls bowled by young fast-bowlers during matches and training sessions to prevent the injuries from workload in fast-bowlers. ${ }^{8}$

In men's cricket, injury surveillance revealed that lumbar spine bone stress injuries and hamstring injuries resulted in most time missed in game due to injuries. ${ }^{9,10}$ The injury surveillance also identified increased workload as a significant risk factor and helped the team management for better workload management and preparation to reduce the injury. ${ }^{10-12}$ In a study conducted by Perera et al. on the Australian elite female cricketers, fast bowlers and fast bowler all-rounders sustained $47.8 \%$ of the injuries sustained by the squad. ${ }^{13}$

It is thus evident from the published literature that fast bowlers, due to their repetitive action, are more predisposed to injury as compared to other players. The kinematic analysis of mixed bowling technique revealed the susceptibility to spinal and back injury when compared to other bowling actions. ${ }^{7}$ However, it is very difficult to measure the musculoskeletal loads during the fast bowling using experimental methods, which are generally invasive in nature. That is where biomechanical modeling using computational musculoskeletal biomechanics has the potential to provide a noninvasive method to predict the musculoskeletal loads during complex movements such as fast bowling in cricket. The knowledge of muscular loading in lower limbs during fast bowling can indirectly provide insight into injury mechanisms and could help in workload management and injury prevention. In the present study, we documented the muscular loads obtained using musculoskeletal modeling in fast-bowlers and tried to understand the loading profiles in the muscles in response to the movement, which in turn could help in injury prevention and rehabilitation.

\section{Materials and Methods}

Four Indian male state-level fast bowlers (age: $19.50 \pm 2.52$ years, height: $174.55 \pm 8.38 \mathrm{~cm}$, weight: $65.82 \pm 10.29 \mathrm{~kg}$ ) with no musculoskeletal injury within past 6 months were chosen. The study was approved by the institutional ethics committee. Written informed consent was obtained from all the participants or their legal representatives. Experimental gait data were collected using the full-body Plug-in-Gait marker protocol. The bowlers performed self-selected warm-up exercises and stretches before the bowling. The bowlers were then instructed to ball 6 deliveries similar to match conditions. The kinematics and kinetics were captured using a Vicon system with 18 infrared cameras and 4 AMTI force platforms. The kinematic and kinetic data were filtered using fourth-order Butterworth filter to remove the noise. The marker data were processed on Vicon Nexus software and imported into OpenSim. Gait 2392 model was used and scaled to match the anthropometry of the bowlers. ${ }^{1,2}$ The inverse kinematics and dynamics were used to predict the internal joint loading during the front foot contact (front foot touchdown to foot off from the ground). Static optimization tool in OpenSim was used to estimate the muscle forces during the front foot contact in fast bowling action.

\section{Results}

The kinetic data from force plates captured the forces exerted by the ground on the body at the foot contact. The vertical ground reaction forces acting on the four athletes is shown in Figure 1. It is evident from the figure that the ground reaction force pattern for all the four athletes are not same.

However, the maximum value (magnitude) of the force being generated is comparable for athletes $A 1$ and $A 3$ and between $A 2$ and A4. This information provides an insight on the loading to which the lower limb is being subjected to during the front foot contact in different fast bowlers. With the knowledge of the material properties of the skeletal system and the information on loading, the incidence of stress concentration can be predicted using principles of mechanics of materials.

Figure 2 represents the forces being induced in the iliacus and psoas muscles as predicted by the musculoskeletal modeling in response to the dynamic movement of the fast bowling. The iliacus and psoas are the strongest flexors of the hip joint and help in the flexion of trunk and unilateral flexion of the trunk. These movements are commonly seen in cricket fast bowling during the delivery phase, i.e., after front foot contact.

Figure 3 represents the activation of gluteus maximus 1, gluteus maximus 2, and gluteus maximus 3 during the front foot contact during the fast bowling. The gluteus maximus is divided into three different sub-categories because of the large attachment area on the bones.

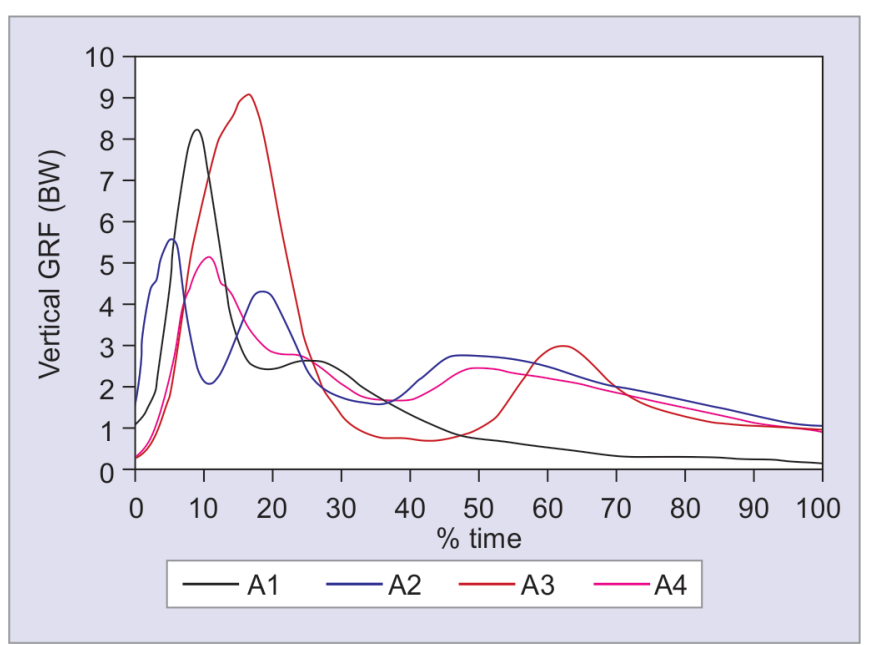

Fig. 1: The vertical ground reaction force component for front foot during fastbowling in terms of body weight, BW $(0 \%$ represents the time of foot contact with the ground and $100 \%$ represents the foot off from the ground). A1, A2, A3 and A4 represent the four athletes respectively 

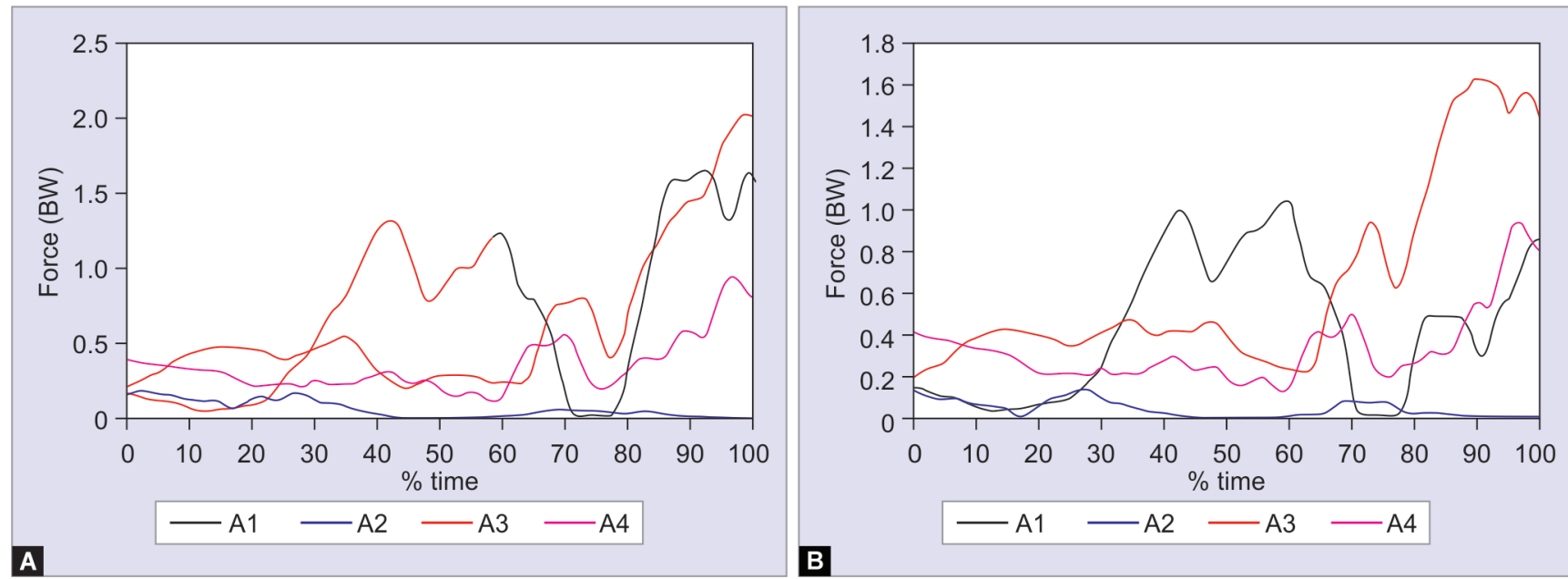

Figs 2A and B: (A) Variation of forces in the iliacus muscle of the four athletes represented in terms of body weight (BW); (B) Variation of forces in the psoas muscle of the four athletes represented in terms of body weight (BW)
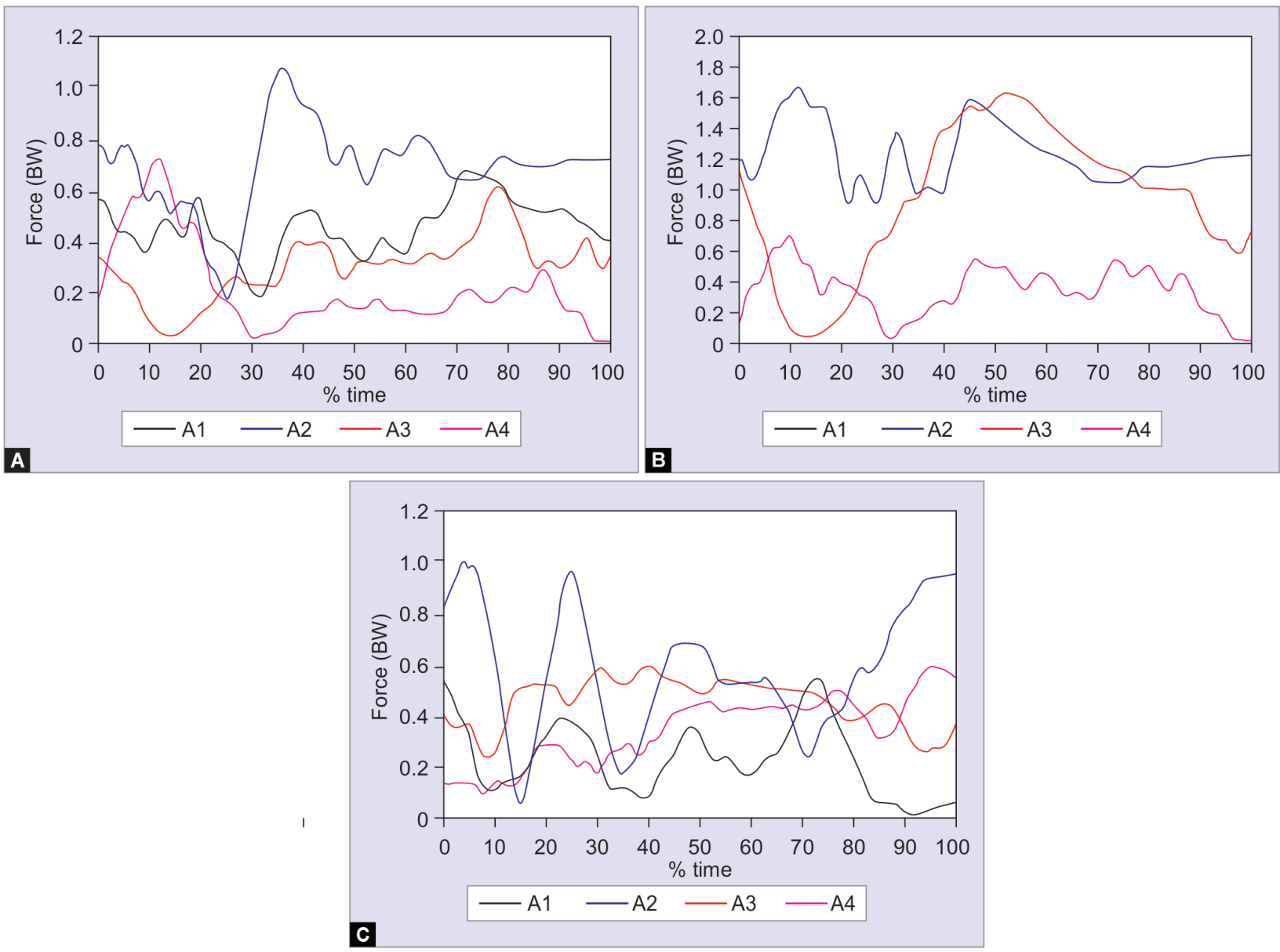

Figs 3 A to C: (A) Variation of forces in the gluteus maximus 1 muscle of the four athletes represented in terms of body weight (BW); (B) Variation of forces in the gluteus maximum 2 muscle of the four athletes represented in terms of body weight (BW); (C) Variation of forces in the gluteus maximum 3 muscle of the four athletes represented in terms of body weight (BW)

The gluteus maximus is mainly responsible for the extension and external rotation of the thigh about hip joint, which movements are a part of the fast-bowling action. The information on the loading profiles of the muscles such as shown in Figures 2 and 3 provides useful insight on the athlete specific muscle activation and loading profiles in response to the dynamic movement under consideration. This information is very useful in understanding the skeletal loading and aid in injury prevention and rehabilitation of the athletes. As an example, we can distinguish the loading patterns of gluteus maximus (1,2 and 3) for athlete A2 (higher) in comparison to other 
athletes. However, in case of iliacus and psoas for athlete $A 2$, the loading is less in comparison to other athletes. Therefore, we can infer that in order to compensate for the reduced activation of iliacus and psoas (flexion or unilateral flexion of the trunk), the athlete $\mathrm{A} 2$ is using compensatory mechanism to perform the movement by externally rotating the thigh to provide the clearance required by the opposite leg. In addition, the ground reaction force profile (Fig. 1) for athlete A2 was different from other athletes and includes a prominent double-humped profile representing loading and unloading in a very small instant of time. This will further affect the activation of the muscles as they are activated in response to the external loading, i.e. ground reaction force. Similarly, for athletes A1 and $A 3$, the increased activation of iliacus and psoas can be related to the high ground reaction forces at the touch down and was further balanced by the increased activation of gluteus maximus 1 and 2 as shown in the Figures 1, 3A and B respectively.

\section{Discussion}

The knowledge of kinetics of the lower body during fast bowling in cricket has the potential to provide us the information on the biomechanical aspects of such a complex movement, with applications ranging from performance enhancement to injury prevention. The proposed methodology utilizes both experimental (motion analysis data) and computational (musculoskeletal modeling) tools to extract the kinetics involved in fast bowling in terms of individual muscle and joint forces. The accuracy of the prediction depends on the accuracy of the experimental data and the consistency of the model being utilized (using the same set of boundary conditions in the computational model) to get the reproducible results. Human movement is variable in nature; it is important to note that even if all the environmental conditions remain the same, there is quantifiable variability in the kinematics and kinetics of movement between individuals. So this inherent variability in the movement can also be expected in the computational results. Therefore, we can expect a reproducible muscle excitation in the athlete-specific simulations within the limits of natural variability in movement. The results thus obtained will further helps us to understand the individual variability in different bowlers with respect to bowling action, skill level, etc. Since the number of athletes included in this study are small, future studies with a large number of athletes with variable backgrounds in terms of skill level, bowling action, etc., are needed to get a better understanding of the dynamics involved during fast bowling. Additionally, we did not capture any electromyographic data to verify the results of the computational model. Therefore, larger studies, with surface electromyographic data to verify the results of computational model for superficial muscles, are needed to have more reliability and confidence on the predicted results.

A number of studies using this computational approach have been documented in the literature for walking, running, jumping, sidestep cutting, and cycling. ${ }^{14-16}$ During a study on sidestep cutting, Maniar et al. reported that vertical ground reaction component was supported by vasti, gluteus maximus, soleus, and gastrocnemius. It was further reported that the same muscles, along with hamstrings, were primarily responsible for anteroposterior ground reaction force (propulsion and braking) ${ }^{14}$ To our knowledge, no previous study has looked at muscle force estimation in cricket fast bowling. Therefore, this is an area for further investigation with large number of athletes and electromyographic data. It is important to appreciate that this knowledge of muscular forces will help us to understand the loading pattern of different muscles and could aid in designing strengthening and rehabilitation protocols by looking at the individual contribution of the muscles under consideration. ${ }^{16,17}$ The proposed athlete-specific approach may result in a better understanding of the individual dynamics during complex movements such as fast bowling and also help achieve better performance with athlete specific inputs from coaching and sports sciences disciplines.

\section{ConClusion}

The present study provides an insight into the lower limb muscular loading in fast bowlers using a noninvasive method and allows an understanding of the muscle dynamics during a complex movement such as fast bowling. This information can provide insights on the role of various muscles during a dynamic event and help sports scientists to understand the optimum force generation and its optimization for improved performance and injury prevention in bowlers. This quantitative information on lower limb loading at various joints can help in designing efficient strengthening and rehabilitation protocol for individual fast-bowler.

\section{Acknowledgments}

This paper was presented by the principal author in WCSMC 2019 Loughborough, UK.

\section{References}

1. Delp SL, Anderson FC, Arnold AS, et al. OpenSim: open-source software to create and analyze dynamic simulations of movement. IEEE Transact Biomed Engineer 2007;54(11):1940-1950. DOI: 10.1109/ TBME.2007.901024.

2. Seth A, Hicks JL, Uchida TK, et al. OpenSim: simulating musculoskeletal dynamics and neuromuscular control to study human and animal movement. PLoS Comput Biol 2018;14(7):e1006223. DOI: 10.1371/ journal.pcbi.1006223.

3. Orchard JW, Kountouris A, Sims K. Incidence and prevalence of elite male cricket injuries using updated consensus definitions. Open Access J Sports Med 2016;7:187. DOI: 10.2147/OAJSM.S117497.

4. Stretch RA. The incidence and nature of injuries in first-league and provincial cricketers. S Afr Med J 1993;83(5):339-342.

5. Stretch RA. The seasonal incidence and nature of injuries in schoolboy cricketers. S Afr Med J 1995;85(11):1182-1184.

6. Leary T, White JA. Acute injury incidence in professional county club cricket players (1985-1995). Br J Sports Med 2000;34(2):145-147. DOI: 10.1136/bjsm.34.2.145.

7. Foster D, John D, Elliott B, et al. Back injuries to fast bowlers in cricket: a prospective study. Br J Sports Med 1989;23(3):150-154. DOI: 10.1136/ bjsm.23.3.150.

8. Gregory $\mathrm{PL}$, Batt $M E$, Wallace WA. Is risk of fast bowling injury in cricketers greatest in those who bowl most? a cohort of young english fast bowlers. Br J Sports Med 2004;38(2):125-128. DOI: 10.1136/ bjsm.2002.000275.

9. Orchard J, James T, Kountouris A, et al. Changes to injury profile (and recommended cricket injury definitions) based on the increased frequency of Twenty20 cricket matches. Open Access J Sports Med 2010;1:63. DOI: 10.2147/OAJSM.S9671.

10. Orchard JW, James T, Portus MR. Injuries to elite male cricketers in Australia over a 10-year period. J Sci Med Sport 2006;9(6):459-467. DOI: 10.1016/j.jsams.2006.05.001.

11. Dennis R, Farhart R, Goumas C, et al. Bowling workload and the risk of injury in elite cricket fast bowlers. J Sci Med Sport 2003;6(3):359-367. DOI: 10.1016/S1440-2440(03)80031-2. 
12. Orchard JW, Blanch P, Paoloni J, et al. Cricket fast bowling workload patterns as risk factors for tendon, muscle, bone and joint injuries. Br J Sports Med 2015;49(16):1064-1068. DOI: 10.1136/ bjsports-2014-093683.

13. Perera NK, Kountouris A, Kemp JL, et al. The incidence, prevalence, nature, severity and mechanisms of injury in elite female cricketers: a prospective cohort study. J Sci Med Sport 2019;22(9):1014-1020. DOI: 10.1016/j.jsams.2019.05.013.

14. Maniar N, Schache AG, Cole MH, et al. Lower-limb muscle function during sidestep cutting. J Biomech 2019;82:186-192. DOI: 10.1016/j. jbiomech.2018.10.021.
15. Lin YC, Kim HJ, Pandy MG. A computationally efficient method for assessing muscle function during human locomotion. Int J Numer Metho Biomed Engineer 2011;27(3):436-449. DOI: 10.1002/ cnm.1396.

16. Buchanan TS, Lloyd DG, Manal K, et al. Neuromusculoskeletal modeling: estimation of muscle forces and joint moments and movements from measurements of neural command. J Appl Biomech 2004;20(4):367-395. DOI: 10.1123/jab.20.4.367.

17. Pandy MG. Computer modeling and simulation of human movement. Annu Rev Biomed Eng 2001;3(1):245-273. DOI: 10.1146/annurev. bioeng.3.1.245. 